\author{
DR ARNOLD BERLINER UND PROF. DR AUGUST PUTTER
}

\begin{tabular}{lll}
\hline Siebenter Jahrgang. & 20. Juni 1919. & Heft 25. \\
\hline \hline
\end{tabular}

\section{Die Grundlagen der Kinematographie ${ }^{1}$ ).} Ton Dr. W. Merté, Jena.

Die vorliegende Darstellung soll in kurzer Zusammenfassung die Grundlagen der Kinematographie behandeln. Zu diesem Zwecke teilen wir unsere Ausführungen in zwei Abschnitte ein, indem wir im ersten von den psychologischen und physiologischen Bedingungen sprechen, die beim Menschen die kinematographischen Täuschungen hervorrufen, und im zweiten Abschnitte von den technisch-physitalischen Einrichtungen und Anordnungen, die geeignet sind, jene Bedingungen herbeizuführen.

\section{Die psychologischen und die physiologischen} Bedingungen der Kinematographie.

Schon längst bekannt ist, daß eine Reihe in sich unbewegter Bi'der, die mehr oder weniger unmittelbar aufeinanderfolgende Bewegungszustände von Personen oder Gegenständen zeigen, den Augen genügend schnell nacheinander dargeboten, unter Umständen den Eindruck eines Bildes sich bewegender Personen oder Gegenstände erweckt. Die Ursache dieser Erscheinung glaubte man ursprïnglich in der schon Ptolemüus bekannten Eigentümlichkeit des Auges gefunden $\mathrm{zu}$ haben, daß die Lichtempfindung eines auf das Auge einwirkenden Lichtreizes bei dessen plötzlicher Unterbrechung längere oder kürzere Zeit braucht, um abzuklingen. - Wir betrachten diese Anschauung, ciie die kinematographischen Gesichtstäuschungen dureh "Nachbildwirkungen" zu erklären sucht; hier als überholt; werden aber trotzdem nachher über die Nachbildwirkung noch Näheres zu sagen haben, da sie für die heute üblichen Kinematographen, wenn auch nicht eine grundlegende, so doch eine sehr wichtige Bedeutung hat.

Um das eigentliche Wesen der kinematographisehen Gesichtstäuschungen zu ergründen, kann man sich nach $P$. F. Linke $e^{2}$ ) am bequemsten des erstmalig im Zeißwerk zu Jena hergestellten Tautoskops bedienen, einer Projektionseinrichtung, ,die das Prinzip der Kinematographie gewissermaßen auf seinen einfachsten Ausdruck bringt". Sie besteht im wesentlichen in der

\footnotetext{
1) Auf Anregung der Schriftleitung gedenke ieh von Zeit zu Zeit über Fragen der 'Kinematographie zu berichten. Der vorliegende Aufsatz gibt einen kurqen Uberblick über die Grundlagen der Kinematographie und dient als Einleitung der beabsichtigten Aufsătze.

i) $P$. $F$. Linke, Grundfragen der Wahrnehmungslehre, Nünchen 1918 , E. Reinhardt, S. 269 ff.
}

Nw. 1919.
Neben- oder. Ubereinanderanordnung zweier gleicher Projektionsapparate, deren Objektive auf dem Schirm möglichst genau dasselbe Bildfeld auszeichnen. Setzt man nun in jeden der beiden Apparate je ein Diapositiv ein, das auf schwarzem Grunde je einen horizontalen bzw. einen um $60^{\circ}$ gegen die Horizontale geneigten, hellen Strich trägt, und projiziert die beiden Striche nacheinander auf den Schirm, so sieht man bei richtiger Wahl des zeitlichen Abstandes der beiden Projektionen den horizontalen Strich eine Drehung um $60^{\circ}$ in die Lage des zweiten Striches ausführen. Der Beobachter sieht also statt der zwei Striche nur einen, der sich eben aus der horizontalen Lage in die zweite um $60^{\circ} \mathrm{zu}$ dieser geneigten dreht, er identifiziert die beiden Striche. Er sieht den momentanen Ortswęchsel des idẹtisch bleibenden Striches, ohne aber die Bewegungsbahn, die Bewegungsphasen des Striches auch bei gespanntester Aufmerksamkeit feststellen zu können. Bringt man nämlich an einer Stelle des Bildfeldes $\mathrm{zw}$ ischen den beiden Endlagen eine dauernd sichtbare, dünne Linie an, über die der Strich bei seiner Drehung hinweggleiten müBte, so sieht man bei der Projektion wohl den Strich seine. Dre-

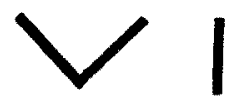

Fig. 1.

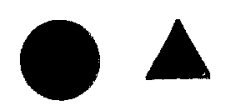

Fig. 2.
Diapositivbilderpaar zum Tautoskopversuch.

hung ausführen, aber jene Linie bleibt in ruhigem Verharren vollkommen unberührt von dem-sich dentlich bewegenden Strich. Dieses Bewegungssehen ohne Bewegungsphasen und ohne bewegtes Objekt ist nach P. F. Linke ein typisches Beispiel für „Umgestaltung auf Grund assimilativer Wahrnehmung". Wie man etwa einer aus Punkten zusammengesetzten Linie in der Wahrnehmung unmittebar die Gestalt des Kreises zuerkennt, obwohl nicht einmal eine geschlossene Kurve vorliegt, so wird in dem Falle unseres Versuches am Tautoskop eine Gegebenheit, der in der'eigentlichen Wahrnehmung die Kriterien der Bewegung fehlen, zwangsläufig als Bewegung vorgestellt, so daß das Vorgeste'lte der Wirklichkeitssuggestion eigentlicher Wahrnehmung teilhaftig wird und hierdurch mit sinnlicher Lebhaftigkeit wahrgenommen wird. Fig 1 zeigt ein weiteres Diapositivbilderpaar. Bei der Projektion der beiden Figuren in angemessenen zeitlichen $\mathrm{Ab}$ ständen sieht man die deutliche Bewegung einer einzigen, nämlich einen Winkel, der zu einer Linie zusammen- und bei entsprechender Fort- 
setzung des Versuches wieder zum Winkel auseinanderklappt. Benutzt man die beiden Diapositivbilder, die Fig. 2 darstellen soll, so kann die tautoskopische Projektion auch dieser einander weniger ähnlichen Figuren den Schein der Bewegung einer Figur hervorrufen. Man sieht dabei die geradlinigen Seiten des Dreiecks sich allmählich umbiegen, bis sie eineu Kreis bilden und umgekehrt. In analoger Weise kann man z. B. auch einen Apfel sich in eine Birne verwandeln lassen.

Werden die beiden ruhenden Bilder, die die Endphasen einer sichtbaren Bewegung darstellen sollen, hinsichtlich der Gestalt oder auch noch des Ortes allzu verschiedenartig gewählt, so wird der Bewegungseindruck gestört. Es werden dann nicht nur qualitativ, sondern auch numerisch verschiodene, d. h. zwei getrennte Gebilde gesehen, nicht mehr eins, das sich verändert oder gegebenenfalls seinen Ort wechselt. Es muB also zwischen den einzelnèn zur bewegten Einheit verschmelzenden Bildern eine Ähnlichkeit bestehen, besonders wohl hinsichtich der räumlichen Eigensehaften (Gestalt, Lage). Die Ähnlichkeit der Farbe hat nach bisher vorliegenden Versuchsergebnissen nur eine sehr geringe Bedeutung. AbschlieBende Untersuchungen, auf Grund deren die zur Identifikationstäuschung notwendige Alnnlichkeit genau bestimmbar wäre, harren noch der Erledigung.

Zur Erzeugung der Identifikationstäuschung oder des sogenannten, stroboskopischen Effektes" ist aber neben der Ähnlichkeit auch oine genügend schnelle Aufeinanderfolge der Bilder notwendig, so dab sie dem Bewubtsein "ein ,jetzt" (in der „psychischen Präsenzzeit") vorliegendes, zusammengehöriges Ganze etwa in demselben Sinne sind, in welchem man dies von den sukziessiven Teilen eines gesprochenen Wortes oder von den Tönen einer Melodie sagen kann. Die Bewegung wird dann unmittelbar wahrgenommien (ähnlich wie beispielsweise die des Sekundenzeigers einer Uhr) und nicht (wie die des Stundenzeigers) auf Grund eines reproduktiven Erinnerungsaktes erschlossen. Man kann also sagen, die Projektionen müssen pausenlos erfolgen, um die stroboskopische Täuschung zu erzielen; wird die Pause zu lang, so kann die Identifizierung nicht mehr eintreten; werden die Bilder gleichzeitig projiziert, so kann auch keine stroboskopische Bewegung gesehon werden; denn Bewegungs- oder Umwandlungsphasen eines Gegenstandes können selbstverständlich nicht gleichzeitig sein.

Nicht unerwähnt mag bleiben, daß es stroboskopische Erscheinungen gibt, die in bloßen Identifikationen bestehen, ohne daB eine Bewegung vorgetäuscht wird. Dieser Fall tritt offenbar bei der Projektion von (hinsichtlich der räumlichen Eigenschaften) völlig gleichartigen Bidern durch ein Tautoskop oder durch einen Kinoprojektor ein. Auch hier ist nur bei pausenloser (aber nicht gleichzeitiger oder in langen Zwi sehenräumen erfolgender) Projektion der Bilder der Eintritt des reinen stroboskopischen Effektes möglich. Die ruhenden Titelüberschriften, die bei kinematographischen Vorführungen mitunter den eirzelnen Szenen vorangehen, sind ein Beispiel solcher stroboskopischen Täuschungen; sie sind, durch hinreichend schnell aufeinanderfolgende Projektionen einer groben Reihe unter sich in jeder Beziehung gleicher Filmbilder dieser Utberschriften zustandegekommen, eine echte Identifikationstäuschung, ohne daß sich mit dieser, eben infolge der Gleichartigheit der zur Einheit identifizierten zahlreichen Bilder, der Eindruck von Bewegung verknüpft.

Bei unseren Versuchen an Tautoskop kann man leicht zeigen, daB die Vor" täuschung der Identität und der Bewegung auch dann noch eintritt, wenn die Projektionen des ersten und zweiten Phasenbildes zeitlich nicht ganz unmittelbar nacheinander erfolgen, sonderr vielmehr beide Gesichtswahrnehmungen durch eine kurze, aber immerhin bemerkbare $Z$ wischen. zeit getrennt sind, in der ein dunkler, über das Bildfeld hinweggleitender Schatten beobachtet werden kann. Damit ist nachgewiesen, daß die. kinematographische Täuschung auch ohne die physiologische Verschmelzung den zugehörigen Netzhautreize (Nachbildwirkung) eintritt, daß also die pausontose Projektion psychologisch (im Sinne der psychischen Präsenzzeit) zu verstehen ist.

Bei den heute üblichen Kinoprojektoren mit ruchweise bewegtem Filmband, über die später noch näher zu hándeln sein wird, spielt gleichwohl jener phýsiologische Verschmelzungsvorgang eine wichtige Rolle. Soweit seine Gesetze für die Kinematographie in Betracht kommen, sollen. diese jetyt hier Erwähnung finden. Bei den ge* nannten Apparaten steht das Filmbild währead der Projektion unbeweglich fest im ,Bildfenster". Die Weiterfortschaltung dieses Bildes und das Eintreten des nächsten Bildes in das Fenster wird, um den Gesamteindruck der Projektion nicht zu stören, durch eine rotierende dunkle Blende verdeckt. Der dadurch bedingte Wechsel von hell und dunkel machte sich bei den älteren Apparaten als ein "Flimmern" sehr unangenehm bemerkbar. Durch empirische Untersuchungen ist man aber dahin gekommen, diese Flimmererscheinung fast vollkommen zu vermeiden. LäBt man nämlich Lichtreize in allmählich immer rascherer Folge auf die Netzhaut einwirken, so gelangt man über die Stadien der Wahrnehmung der Einzelreize und des Flimmerns hinweg zu dem Stadium der Verschmelzung, bei dem ein ganz stetiger Lichteindruck erzeugt wird. Die für die Verschmelzung notwendige Anzahl der Lichtwechsel in der Sekunde ist durch die ,Verschmelzungsfrequenz" gegeben. Die meisten Gesetze der Verschmelzungsfrequenz kann man bequem mit rotierenden Kreisscheiben, auf denen sich schwarze 
bzw. weiBe Sektoren befinden, ermitteln. Da es nach Untersuchungen von Baader.1) für die Verschmelzung belanglos ist, ob der Lichtwechsel wie bei der kinematographischen Projektion in dem gauzen beobachteten Feld gleichzeitig erfolgt, oder wie bei der Beobachtung jener rotieyenden Scheiben die Grenze zwischen hell und dunkel über die Netzhaut hinläuft, so können die mit diesen Scheiben gewonnenen Ergebnisse unmittelbar auch auf die Projektionen durch Kinom apparate mit ruckweise bewegtem Filmband angewandt werden. Der einfachste Fall ist offenbar der, bei dem eine Scheibe mit je einem gleich großen weißen und schwarzen Sektor (vgl. Fig. 3) benutzt wird, d. h. der Fall, in dem die Einwirkungs- und Unterbrechungszeiten des Lichts gleich lange dauern. Es läßt sich leicht feststellen, daß für diesen Fall, in dem das sogenannte ,Sektorenverhältnis" den Wert 1 besitzt, die Verschmelzungsfrequenz mit steigender Intensität des intermittierend einwirkenden Lichtes zunimmt. Die bisher überhaupt beobachteten Werte für die Verschmelzungsfrequenz liegen etwa zwischen 10 und 70 pro Sekunde. T.C. Porter ${ }^{2}$ ) ist es sogar gelungen, für das Sektorenverhältnis 1 die Abhängigkeit der Verschmelzungsfrequenz von der Lichtstärke mathematisch zu formulieren. Für die aus hygienischen Gründen günstigste

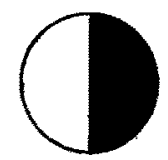

Fig. 3. Scheibe zur Bestimmung der Verschmelzungsfrequenz. Sektorenverhälnis $1: 1$.

Beleuchtungsstärke des Projektionsschirmes von 50 Meterkerzen ergibt dio Rechnung dann eine Verschmelzungsfrequenz zu 46,8. Nimmt man als untere Grenze der noch zulässigen Beleuchtungsstärke etwa 10 Meterkerzen an, so berechnet sich hierfür die Verschmelzungsfrequenz zu 38. Die modernen Kinoprojektoren erreichen nicht einmal diese untere Grenze der Verschmelzungs. frequenz (die übliche Zahl der Bildwechsel pro Sekunde beträgt nämlich bei ihnen etwa 20), da wegen der beschränkten Festigkeit von Film und Meehanismus die Geschwindigkeit der Bildfolge eine gewisse Größe nicht überschreiten darf. Durch Variierung des Sektorenverhältnisses kann man nun aber die Verschmelzungsfrequenz nicht unerheblich herabsetzen und damit der praktischen Erfüllung der theoretischen Forderung

1) W. Nagel, Handb. d. Physiologie d. Menechen, Braunschweig, Friedrich Vieweg \& Sohn, III. Bd., S. 256 .

2) T. C. Porter, Contributions to the study of Flicker in Proceedings of the Royal Society of London, September 1902, S. 313 ff.

3) H. Lehmann,-Die Kinematographie, Bd. $358 \mathrm{~d}$. Sammlung ,Aus Natur und Geisteswelt", B. G. Teubner, Leipzig 1911, S. 29. näher kommen. Marbe $e^{1}$ hat, um zu numerischen Angaben za gelangen, ein feststehendes Diapositiv projiziert, durch rotierende Scheiben, die der Reihe nach nebenstehend zur Darstellung gebracht sind (vgl. Fig. 4-8), den Strahlengang der Projektionsanordnung periodisch unterbrochen und die, kritische Periodendauer", aus der sich die Verschme'zungsfrequenz unschwer berechnen läßt, bestimmt. Die folgende Zusammenstellung 1, die aus den Mittelwerten zweier von Marbe angegebenen Versuchsreihen gewonnen ist,

Zusammenstellung 1 .

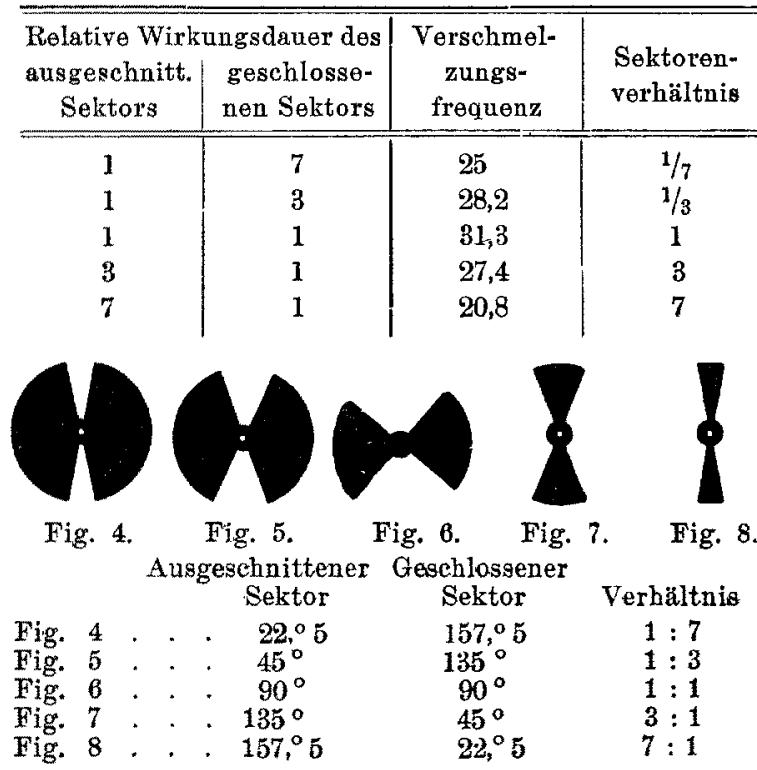

zeigt, daß für das Sektorenverhältnis 1 die Verschmelzungsfrequenz ein Maximum ist. Die Marbeschen Resultate sind zwar bei verhältnismäBig geringer Beleuchtungsstärke (die Lichtquelle war eine Giühlampe) ermittelt worden, und vermutlich ist die Wirkung des Sektorenverhältnisses von dieser nicht ganz unabhängig; seine Messungen stimmen aber gut mit den Erfahrungen, die man bei der in der Regel bedeutend lichtstärkeren kinematographischen Projektion gemacht hat, überein.

Wählt man nun eine Blendenscheibe, deren Sektorenverhältnis gröber als 1 ist, so erreicht man neben der erwünschten Verringerung der Verschmelzungsfrequenz noch eine bessere Lichtausbeute, da ja die mittlere Helligkeit nach der unter dem Namen des Talbotschen Gesetzes bekannten Regel mit wachsendem Sektorenverhältnis offenbar gröBer werden muß. Diese Erkenntnisse für unsere Kinoprojektoren angewandt, würden verlangen, daß man die Ruhestellung des Films, in der die Exposition des Bildes stattfindet, möglichst lang dauernd läßt im Verhältnis zu der Zeit, in der der Film. weiter geschaltet wird. Aber auch hier sind Grenzen gesetzt, da mit ab-

1) K. Marbe, Theorie der kinematographischen Projektionen, J. A. Barth, Leipzig 1910, S. 50 ff. 
nehmender Filmtransportzeit Film und Mechanismus immer stärker beansprucht werden. Aus diesem Grunde wird ein Sektorenverhältnis von 5 kaum überschritten.

Um das Flimmern in noch höherem Grade unschädlich zu machen, hat man daher schon lange noch andere Mittel in Anwendung gebracht. So hat man z. B. den Sektor, der die Bildfortschaltung verdeckt, mit Schlitzen oder Löchern versehen, aus Mattglas angefertigt, oder auf ähnliche Weise die Verdunklung aufgehe'lt, natürlich immer so, daß die Bewegung des Filmbandes nicht bemerkt werden kann. Bei solehen Anordnungen hebt sich das Bild gewissermaßen. nur von einer halbdunklen Wand ab, es erscheint daher lichtsèhwächer und auch flauer. Wichtiger als diese Verringerung des Flimmerns durch Herabsetzung der Reizschwankungen ist die durch Einschallung überzähliger Verdunklungen herbeigeführte Abschwächung der Flimmererscheinung. Solche Verdunklungen kann man z. B. dadurch erreichen, daß man der Blendenscheibe neben dem eigentlichen Abdecksektor, der den Bildwechsel unsichtbar macht, noch weitere dunkle Sektoren einfügt, die während der Ruhestellung des Bildes im Fenster den Strahlengang unterbrechen. Zwar geht selbstverständlich hierdurch Licht verloren, aber durch die Anwendung derartiger mehrflügliger Blenden läßt sich das Flimmern vollkommen unterdrücken. Beispielsweise sind nach Ergebnissen aus Versuchen Marbes ${ }^{1}$ ) an Scheiben, die mit schwarzen und weißen Sektoren entsprechend den nebenstehenden Fig. 9-14 versehen waren, die Verschmelzungsfrequenzen $n$ berechnet und in Zusammenstellung 2 angegeben.

Zusammenstellung 2.

\begin{tabular}{c|c|c|c}
\hline Scheibe nach & $n$ & Scheibe nach & $n$ \\
\hline Fig. 9 & 35,46 & Fig. 12 & 20,24 \\
Fig. 10 & 32,47 & Fig. 13 & 21,83 \\
Fig. 11 & 35,97 & Fig. 14 & 23,70
\end{tabular}

gestört, als wenn man die gleichen periodisch unterbrochenen Lichterscheinungen in einem hellen Raume beobachten würde. Andere hier nicht erwähnte Eigenschaften, Regeln oder Gesetze der Verschmelzungsfrequenz sind für unser vorliegendes Problem weniger von Belang.

Die durch den stroboskopischen Effekt vorgetäuschten Bewegungen erfolgen nach ganz bestimmten Prinzipien. So spricht Linke von dem "Prinzip des kürzesten Wahrnehmungsweges". Dieses besagt, daB in der Regel die stroboskopische Bewegung in der Richtung des küurzesten Weges zustande kommt. Zur näheren Erläuterung brauchen wir nur unseren ersten Versuch am Tautoskop etwas zu variiren. Ne'tenen wir an, dab die Diapositivbi'der der beiden Endphasen nicht zwei um $60^{\circ}$ gegeneinander geneigte Striche darstellen, sondern vielmehr zwei Striche, die einen Winkel, der größer als $90^{\circ}$ ist, miteinander einschlieBen, so ergibt den kürzesten Weg von der Lage des ersten Striches in die des zweiten nicht mehr die Drehung um die Winkelgröße, sondern die erwähnte Regel bestimmt dann eine andere Art der Bewegung, beispielsweise ein Gleiten aus der einen in die andere Lage. Eine Folge dieses

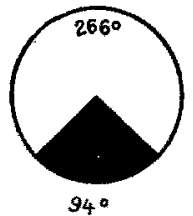

Fig. 9.

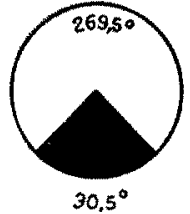

Fig. 10.

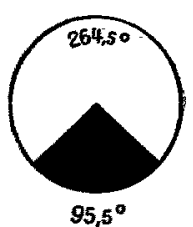

Fig. 11.

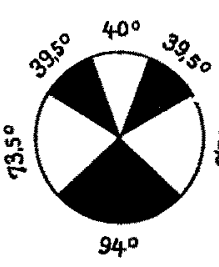

Fig. 12.

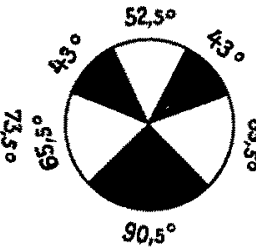

Fig. 13.

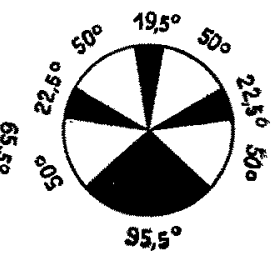

Fig. 14.
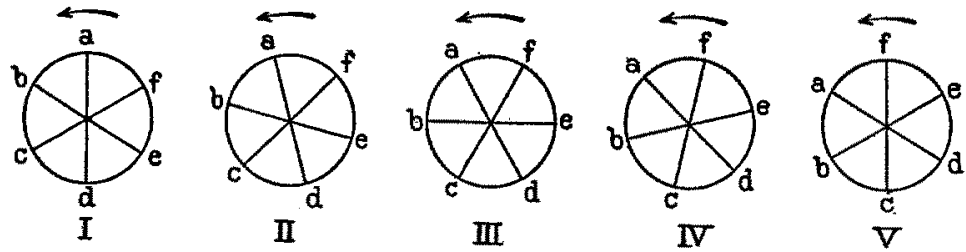

Fig. 15. Figuren zur Erklärung des „Radphbìnomens".

In bemerkenswerter Weise sind die Flimmererscheinungen vom Adaptationszustand des Auges abhängig. Bei genügend hohen Lichtstärken (bei sehr kleinen liegen die Verhältnisse allerdings anders) nimmt die Verschmelzungsfrequenz mit zunehmender Dunkeladaptation ab. Man wird also im allgemeinen nach hinreichend langem Verweilen im Dunkeln durch das Flimmern weniger

1) Vergl, o, S. 43 ff.
Sachverhaltes ist es auch, daß unter Umständen stroboskopische Bewegungen vorgetäuscht werden, die dem objektiven Bewegungsvorgang; dessen Phașenbilder beispielsweise mit einem Kinoaufnahmeapparat gewonnen seien, gar nicht entsprechen. Häufig kommt eine solche Vortăuschung falscher Bewegungen bei der kinematographischen Darstellung fahrender Wagen zustande. An Hand der obenstehenden Fig. 15 ist die Erklärung dieser Erscheinung leicht. Es 
seien durch Fig. I und V zwei aufeinanderfolgende Aufnahmen des Rades dargestellt, d. h. zwischen je zwei Aufnahmen hat das Rad sich um genau einen Speichenwinkel gedreht; das Pad scheint, da alle Speichen der Form und GröBe nach völlig gleich sind und deswegen sämtlich ohne weiteres miteinander identifiziert werden können, stillzustehen. Hat sich dagegen das Rad zwischen je zwei Aufnahmen um einen Winkel, der kleiner bzw. größer als ein haiber, aber immer noch kleiner als ein ganzer Speichenwinkel ist. weitergedreht, so wird auf Grund des Prinzips des kürzesten "Wahrnehmungsweges eine Vorwärts- bzw. Rückwärtsdrehung durch Tdent: fikation vorgetäuscht. Fig. I und IT bzw. I und IV stellen diese beiden Falle dar. Hat sich schlieblich entsprechend der Fig. I und III das Rad zwischen je zwei Aufnahmen immer gerade um genau einen halben Speichenwinkel gedreht. so führt offenbar das Prinzip des kürzesten Wahrnehmungsweges $z u$ einer Unbestimmtheit. Man kann da ein Vorwärts- oder Rückwärtsdrehen oder ein Pendeln des Rades um seine Achse sehen, je a ach der Autosuggestion des Beobachters; bei hinreichend hoher Bildwechselfrequenz aber scheint las Rad stillzustehen und die Anzahl der Speichen ist scheinbar verdoppelt.

Wählt man für die Projektion eine andere Geschwindigkeit des Films, als man bei der Aufnahme anwandte, so erhält man eine bezüglich der Geschwindigkeit, falsche* Bewegung vorgetäuscht. Man kann auf diese Weise z. B. Blüten sich innerhalb weniger Minuten entfalten oder Geschosse langsam ihre Bahn ziehen sehen. Solche Anordnungen, bei denen die Aufnahmegeschwindigkeit (die Aufnahme findet in diesen Fallen meist mit Spezialapparaten statt) wesentlich von der Projektionsgeschwindigkeit abweicht, können wichtige wissenschaftliche und technische Einblicke gewähren. In dieser Hinsicht bekannt geworden ist das $Z$ eitmikroshop von Lehmann ${ }^{1}$, das Bewegungsvorgänge bemerkbar macht, die unserer natürlichen Wahrnehmungsfähigkeit wegen ihres schnellen Verlaufes entgehen würden.

2. Die technische Herbeiführung der psycho-

logischen und physiologischen Bedingungen der Kinematographie.

Zur Herbeifuhrung der Bedingungen, durch deren Erfullung die stroboskopischen Täuschungen erzeugt werden, sind Apparate mannigfachster Art ersonnen worden; ja, diese Täuschungen kommen sogar frei in der Natur vor. In dem engen Rahmen unserer Betrachtungen genügt es, nur kurz bei den älteren Apparaten zu verweilen, um dann über die neuzeitlichen kinematographischen Anordnungen und Einrichtungen wenigstens das Wesentlichste sagen zu können.

Etwa mit dem zweiten Drittel des vorigen Jahrhunderts begann die Tatsache der strobo-

1) Ausgeführt von den Ernemann-Werken A.G.G. Dresden. skopischen Bewegungstäuschungen allgemeineres Interesse $z u$ gewinnen. Neben der Anwendung stroboskopischer Apparate für die verschiedensten Gebiete experimenteller Forschung ging man bald dazu über, diese Apparate auch Zwecken der Unterhaltung dienstbar zu machen. Das sogenannte Lebensrad oder Phänakistoskop ist einer d'er einfachsten und ältesten ,,kinematoskopischen“ Apparate. Es wurde fast gleichzeitig von Plateau und Stampfer erfunden. Das Lebensrad besteht aus einer Kreisscheibe, die um eine durch ihren Mittelpunkt gehende, zu ihrer Ebene senkrechte Achse drehbar ist. Auf einem za dieser konzentrischen Ring sind die Bilder der Bewegungsphasen eines Gegenstandes angebracht, und zwar so, daB zeitlich aufeinanderfolgende Phasen auch räumlich auf der Scheibe aufeinanderfolgen.

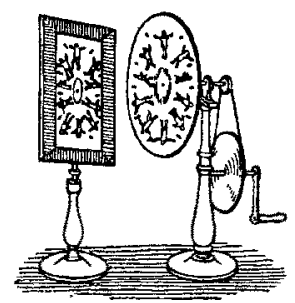

Fig. 16. Das Lebensrad.

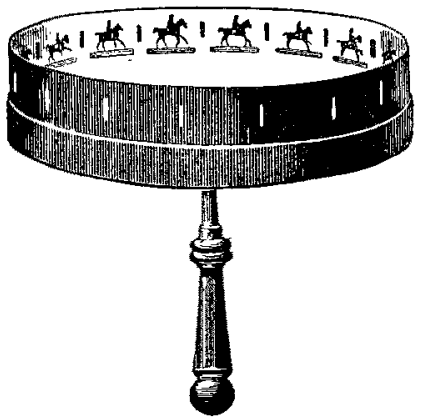

Fig, 17. Anschütz' Schnellseher.

Über oder neben diesen Bildern befindet sich ein Spalt in radialer Richtung. Kehrt man die die Bilder tragende. Seite der Scheibe einem Planspiegel zu, dreht die Scheibe mit thinreichender Geschwindigkeit und sieht durch die Spalte nach dem Spiegel, so erscheint unter bzw. neben den ruhenden Spalten der Gegenstand in Bewegung. Fig. 16 bringt das geschilderte Lebensrad zur Darstellung.

Außer den scheibenförmigen Vorrichtungen führten sich auch zylindrische Apparate ein. Ein solcher ist Anschütz' Schnellseher, der in Fig. 17 abgebildet ist. Hier befinden sich die Phasenbilder eines bewegten Gegenstandes auf der Innenseite eines Hohlzylinders, der um seine Achse drehbar ist. Neben oder uber den Bildern sind achsenparallele Schlitze angebracht. Durch diese kann man auf die gegenüberliegende innere Seite des Zylinders sehen, bei dessen genügend schneller Drehung 
die Phasenbilder zur stroboskopischen Bewegung „verschmelzen". Diese einfachsten Formen von stroboskopischen Apparaten sind nach den verschiedensten Richtungen hin verbessert und sogar mit Projektionsein richtungen versehen worden, um die lebenden Bilder auch einem gröBeren Kreis von Zuschauern gleichzeitig vorzuführen. Sie alle haben den Nachteil, daß nur solche Vorgänge mit ihnen gezeigt werden können, die sich durch eine geringe Zahl von Teilbildern darstellen lassen, und bei denen sich die Bewegungen periodisch wiederholen, so dab also die Endphase der Bilderreihe sich an die Anfangsphase unmittelbar anschließt. Für die Untersuchung vieler physikalischer Erscheinungen leisten sie aber ausreichende Dienste.

Von der größten Bedeutung für die weitere Vervollkommnung der Kinematographie sind die Versuche geworden, auf photographischem Wege die Phasenbilder zu gewinnen. Die ersten kinematographischen Aufnahmeapparate, die die Momentphotographie benutzten, besaßen meist für jedes Bildfeld ein besonderes Objektiv. Mit diesen älteren photographischen Anordnungen war man daher auf eine verhältnismäßig geringe Bilderzahl beschränkt, und viele hatten den Nachteil, daß die mit ihnen erhaltenen Teilbilder stereoskopische Abweichungen zeigten. Einen gröBeren Fortschritt bedeutete daher die ,Mareysche Flinte" Bei dieser ist in den Schaft einer Flinte, in deren Laufmündung sich ein $O b$ jektiv befindet, an Stelle des Schlosses ein Bewegungsmechanismus and eine photographische Platte eingesetzt. Durch den Abzug der Flinte läBt sich mittelst eines Uhrwerkes der Bewegungsmechanismus in Tätigkeit setzen, der die Platte ruckweise derart um eine zu ihrer Ebene senkrechte Achse dreht, daB sie innerhalb einer Sekunde eine volle .Umdrehung zurücklegt und dabei zwölfmal stillsteht. Während des Stillstandes erfolgt jedesmal eine Aufnahme. Marey studierte seit etwa 1882 mit diesem Apparat die Flugbewegung der Vögel. War die Bilderzahl auch noch beschränkt, so war die Mareysche Flinte doch schon ein recht leistungsfähiger, verhältnismäBig einfacher Aufnahmeapparat. Durch Ersatz der photographischen Glasplatte durch lange Bänder aus Papier, dio die lichtempfindliche Schicht trugen, und die durch einen Mechanismus ruckweise von einer Rolle ab- und auf eine zweite aufgewickelt wurden, konnte Marey die Bilderzahl fast beliebig erhöhen. Zwischen den beiden Rollen wurde der ,Film" während des Stillstandes durch ein Objektiv belichtet, das bei der Bewegung des Papierbandes jedesmal abgeblendet wurde. Im Jahire 1889 schlägt Friese-Green als Bildträger den Zelluloidfilm vor, und damit beginnt die Entwicklung des eigentlichen modernen Kirematographen.

Schon aus unseren bisherigen Erörterungen äber die Vorläufer der neuzeitlichen Kinoapparate erkennen wir, daB die physikalisch-technischen
Vorrichtungen, die die Vorführung lebender Bìder ermöglichen, sich in zwei Gruppen teilen lassen, nämlich in die Einrichtungen, die bestimmt sind, geeignete Phasenbilder $z u$ gewinnen, und dann in die Anordnungen, mit deren Hilfe diese als lebende Bilder gezeigt werden können. Die hier in Betracht kommenden Apparate sind heute in den weitaus überwiegenden Fällen neben dem nötigen Zubehör einmal die Kino-Aufnahmekammer und ferner der Kino-Projektor. Die zahlreichen Spezialkonstruktionen, besonders für wissenschaftliche und technische Zwecke, werden hier nicht berücksichtigt werden. Diese beiden Apparate, Aufnahmekammer und Projektor, haben Verschiedenes gemeinsam, z. B. den Film und einen Mechanismus, der den Film bewegt.

Der Film besteht aus einem Zelluloidband vor $35 \mathrm{~mm}$ Breite und oft mehr als $100 \mathrm{~m}$ Länge. Da das Zelluloid sehr feuergefährlich ist, wird der Film neuerdings auch aus Zellit hergestellt, einem Fabrikat der Elberfelder Firma Bayer \& Co., das nur schwer brennbar ist. Die einzelnen Filmbilder sind $18 \times 24 \mathrm{qmm}$ grob; am Rand ist der Film mit einer Perforation versehen, und zwar kommen auf das Bild 4 Löcher auf jeder Seite, in die der Fortbewegungsmechanismus eingreift. Die Maße des Films sind durch internationales Übereinkommen so gewählt, daß jeder Film in jeden Apparat paBt. Das Filmband wird durch eine Antriebsvorrichtung von einer Vorratsrolle auf eine Aufnahmerolle gewickelt und dabei an dem Bildfenster, in dem der Film der Belichtung (Aufnahme) bzw. der Beleuchtung (Projektion) ausgesetzt wird, vorbeigeführt. Während der Belichtung (Beleuchtung) muB offenbar der im Bildfenster befindliche Teil des Films zum Stillstand gebracht sein. Man hat dafür zwei Lösungsarten gefunden, eine mechanische und eine optische, d. h. entweder bleibt der betreffende Teil des Filmbandes tatsächlich für kurze Zeit im Fenster in Ruhe stehen, oder aber die Bewegung des Filmbandes wird optisch aufgehoben, die kontinuierliche Bewegung wird ,optisch stationär" gemacht. Von diesem Gesichtspunkte aus würden die kinematographischen Apparate (Aufnahmekammer und Projektor) in zwei Grup pen einzuteilen sein:

1. diejenigen, bei denen der Film im Fenster absatzweise fortgeschaltet wird;

2. diejenigen, bei denen das Bildband kontinuierlich durch das Filmfenster hindurchbewegt wird.

Die in der Praxis üblichen Apparate gehören heute noch fast ausschließlich zu der ersten Gruppe. Die nebenstehende Fig. 18 zeigt schematisch die Filmführung in einem Kinoapparate; auf Besonderheiten, wie sie sich an den Modellen der verschiedenen Fabriken finden, wird hier selbstverständlich nicht Rücksicht genommen. Durch die mit gleichförmiger Geschwindigkeit gedrehte Zahntrommel $B$ (Vorwickler) wird das Filmband von der Vorratsspule $A$ abgewickelt 
and dem Bildfenster $D$ zugeführt. Dabei bauscht sich der Film, solange er im Fenster $D$ stillsteht, zwischen $B$ und $D$ zu einer Schleife $C$. Hinter dem Fenster $D$ befindet sich eine Zahntrommel $E$, die das im Fenster befindliche Filmbild ruckweise weiterreiBt; dadurch wird die Schleife $C$ aufgezehrt, und ein neues Bild tritt in das Fenster ein. Hinter der Trommel $E$ bildet sich ein.zweiter Bausch $G$. Dieser wird durch die stetig gedrehte Trommel $F$ aufgebrancht und der Film auf die Spule $I I$ aufgewickelt. Durch. Wiederholung des geschilderten Vorganges wird Bild für Bild, nachdem es kurze Zeit im Fenster stillgestanden hat, ruckweise aus diesem weitertransportiert. Die Bewegung der Zahntrommel $E$ erfolgt durch eine Malteserkreuz-Einrichtung ${ }^{\mathbf{1}}$ ) (andere Mechanis.

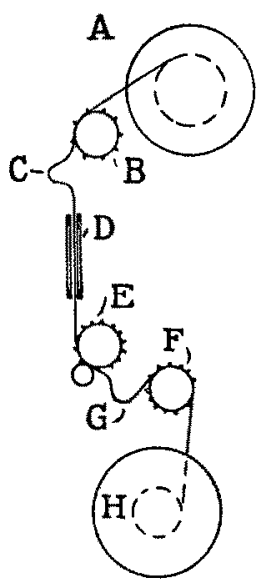

Fig. 18. Schema der Filmbandführung durch einen Kinoapparat mit ruckweise bewegtem Film.

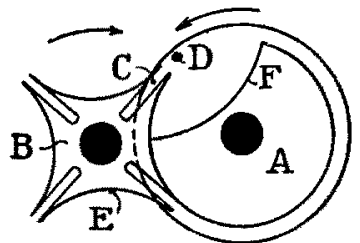

Fig. 19. Schema des Maltesergesperres.

men zur absatzweisen Fortschaltung des Filmbandes, wie ,Greifer", der sich häufig bei Aufnahmeapparaten findet, "Schläger" und ,Reibungsscheiben" seien hier nur dem Namen nach erwähnt). Eine solche zeigt schematisch Fig. 19. Das eigentiche Malteserkreuz $B$ sitzt auf der Achse der Zahntrommel $E$ (s. Fig. 18), deren Zähne in die Perforationen an den Filmrändern eingreifen, und ist mit dieser fest verbunden. Eine Scheibe $A$ ist mit einer größeren, mit einem Stift oder Einzahn $D$ versehenen Scheibe auf einer gemeinschaftlichen Achse befestigt. Wird nun die Achse, auf der diese beiden Scheiben sitzen, gedreht, so kann das Malteserkreuzrad $B$ und mit, ihm die Trommel-E

1) Eine eingehendere Theorie des Malteserkreuzes gibt $C$. Forch auf S. 15 ff. seines Buches: Der Kinematograph und das sich bewegende Bild, Wien und Leipzig, A. Hartlebens Verlag, 1913. (s. Fig. 18) sich nicht bewegen, solange die Scheibe $A$ an dem Bogenstück $E$ des Malteserkreuzes anliegt. Sobald aber der Stift $D$ in einen der Schlitze $C$ des Kreuzrades eingreift, wird dieses unter Einwirkung des Einzahnes $D$ ge dreht. wobei die Aussparung $F$ in der Soheibe $A$ den Vorbeigang der vorspringenden Spitzen des Schlitzes $C$ gestattet; der Film wird dabei durch dio Zahntrommel $E$ (s. Fig. 18) um eine Bildhöhe weitergoschaltet. Bei der in Fig. 19 dargestellten Einrichtung sind 4 Umdrehungen des Einzahnrades notwendig, um das Kreuzrad einmal herumzubewegen. Ist der Umfang der Trommel $E$ (s. Fig. 18) so gewählt, daß. er gleich der Summe der Höhen von 4 Filmbildern ist," so wird bei einer Vierteldrehung dieser Trommel das Filmband um genau ein Bid ruckweise weiterbewegt, Die Malteserkreuzrad-Finrichtung kann gegenüber der in Fig. 19 dargestellten auch auf die verschiedenste Weise modifiziert werden. Es gibt z. B. Ausführungen, bei denen viel mehr als 4 Umdrehungen des stifttragenden Rades notwendig sind, um eine volle Umdrehung des $K_{r e u z}$ rades herbeizufähren.

Nach den Erörterungen des ersten Abschnittes werden bei der Projektion, wenn die Zeit der Ruhestellung des Films im Fenster im Verhältnis zur Zeit der Fortschaltung, des Ruckes, mög'ichst lang ist, zwei Vorteile erreicht; einmal wird das Licht besser ausgenutzt, und dann wird auch das Flimmern wesentlich herabgesetzt. Durch bestimmte Wahl der Abmessungen von Kreuzrad und Einzahnrad kann man die Transportzeit herabsetzen; eine Grenze ist ja aber durch die Festigkeit von Film und Mechanismus gesetzt.

Die Aufgaben, die die Kinoapparate mit ruckweise bewegtem Filmband zu lösen haben, sind, kurz zusammengefaBt, folgende:

Der Mechanismus muB den Film genau um eine Bildbreite weiterschalten und ein ruhiges "Stehen" des Bildes bewirken; die Transportzeit ist auf ein Mindestmaß zu bringen bei möglichster Schonung von Film und Apparat. Diese Forderungen sind bei den modernen Kinoapparaten im allgemeinen genügend erfüllt. Ein Nachteil aller Systeme mit absatzweiser Filmfortschaltung ist die verhätnismäbig geringe Bildwechselfrequenz, die durch die Beanspruchung des Materials bedingt ist: insbesondere bei den Projektionen ist ferner der Lichtverlust durch die rotierenden Blenden, die den Bildtransport zu verdecken und auch meist das Flimmern zu verringern haben, prinzipiell nicht zu vermeiden.

Diese Nachteile können bei den Apparaten mit stetig bewegtem Filmband beseitigt werden. Bei ihnen bewegt sich auch durch das Bildfenster der Film mit geichförmiger Geschwindigkeit. Sowohl für die Aufnahme wie fur die Projektion ist es notwendig, diese Wanderung des Films im Fenster zu kompensieren. Das wird bei den nun zu be- 
sprechenden Anordnungen durch optische Hilfsmittel erreicht, und zwar unterscheiden wir hier drei Möglichkeiten. Die Filmbewegung im Fenster kann optisch aufgehoben werden:

1. durch ein oder mehrere bewegte Objektive,

2. durch Einschaltung von katoptrischen Ausgleichssystemen in den abbildenden Strahlengang,

3. durch Einschaltung von dioptrischen Ausgleichssystemen in den abbildenden Strahlengang.

Von den unter 1. genannten Systemen seien hier die Ausführungsformen erwähnt, bei denen sich ein Objektiv geradlinig und parallel, immer in der gleichen räumlichen Anordnung zum zugehörigen Film bewegt. Bei der Aufnahme sehr weit entfernter Gegenstände odex der Projektion auf einen in größerer Entfernung liegenden Schirm ist, da dann der ReduktionsmaBstab bzw. die Vergrößerung (der entsprechende Wert sei mit $n$ bezeichnet) sehr groß ist, die Wanderung von Film und Objektiv praktisch ohne Bedeutung. Will man mit nur einem Objektiv auskommen, so kann man das Objektiv in einer Schlittenführung parallel zum Filmbild mit diesem wandern lassen,

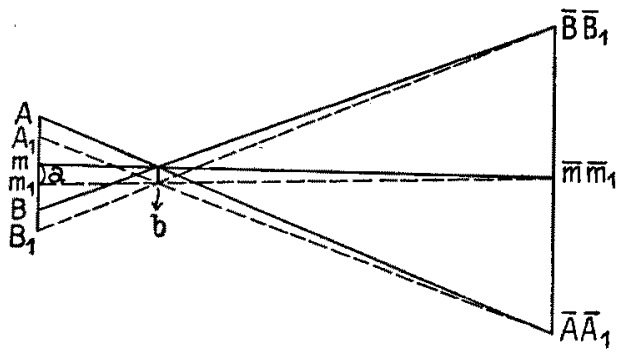

Fig. 20. Sehematisehe Darstellung des Abbildungsvorganges durch ein um die Strecke $b$ geradlinilg und parallel zum Film gewandertes Objektiv. Dieser ist aus der Stellung $A B$ um die. Strecke $a$ in die Stellung $A_{1} B_{1}$ weitergeführt. Die überstrichenen Buchstaben stellen die Bilder der entsprechenden, nicht iberstrichenen Buchstaben vor.

um dann, wenn das nächste Bild zur Abbildung gelangen soll, das Objektiv in die Ausgangsstellung zurückzuführen und das gleiche Spiel zu wiederholen. Besser als solche Apparate mit einem oszillierenden Objektiv sind die Ausführungsformen, bei denen sich eine Reihe von Objektiven in kreisförmiger Anordnung etwa auf einer rotierenden Scheibe befinden. Da dann aber die Objektive nicht genau geradlinig wandern, ist bei höheren Ansprüchen Vorsorge zu treffen, den sich daraus ergebenden Fehler unschädlich zu machea. Die nebenstehende Fig. 20 zeigt schematisch den Abbildungsvorgang beim optischen Ausgleich durch ein geradlinig wanderndes Objektiv. Bezeichnet man mit $a$ die Verschiebung des Filmbildes und mit $b$ die des Objektivs, so muß nach den Abbildungsgesetzen

$$
b=a \cdot \frac{1+n}{2+n+\frac{1}{n}}
$$

sein, um den Ausgleich herbeizuführen. Für $n=\infty$ wird in der Tat $b=a$.

Das Prinzip des optischen Ausgleichs der Bildwanderung durch einen bewegten Spiegel ist schon lange, bevor man an Kinematographen mit stetig bewegtem Filmband dachte, beim Heliostaten angewandt worden. Auch von den katoptrischen Systemen, die das wandernde Filmbild optisch stationär machen sollen, sind die verschiedensten Ausführungsformen angegeben worden. Sie benutzen je nach den Verhältnissen eine geringe oder grobe Anzahl von Spiegeln, die oszillierende oder rotierende Bewegungen ausführen. Fig. 21 zeigt schematisch eine Anordnung, bei der durch eine stetig gedrehte prisma-

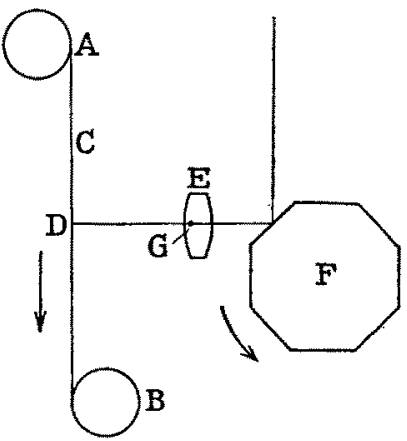

Fig. 21. Sehematische Darstellung eines optischen Ausgleichssystemes, das aus einer vor dem Objektiv befindlichen, rotierenden Spiegeltrommel besteht.

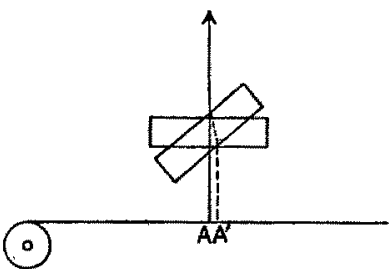

Fig. 22. Schematische Darstellung des optischen Ausgleiches durch Drehung einer Planparallelplatte.

tische Linsentrommel $F$, deren Seitenwände von Planspiegeln gebildet werden, der Ausgleich erfolgt. Der Film $C$ wickelt sich von der Rolle $A$ mit gieichförmiger Geschwindigkeit ab und auf die Rolle $B$ auf. $E$ ist das Objektiv, und $D$ sei die Mitte eines Filmbildes, das sich in der Zeichnung gerade in der Stellung befindet, wo der Hauptstrahl des abbildenden Strahlenbüschels für $D$ mit der optischen Achse von $E$ zusammenfällt. Je ein Filmbild und ein Spiegel der Trommel, deren Querschnitt ein regelmäBiges Vieleck ist, treten bei. der Bowegung stets miteinander in Wechselbezichung, um immer voñ dem nâchsten Elementenpaar abgelöst $z u$ werden. Ist $G$ der dem Film zugekehrte Hauptpunkt des Objektivs $E$, bezeichnet man $G D$ mit $f$ und die halbe Bildhöhe mit $h$, so ist der halbe Bildwinkel $w$ gegeben durch: tang $w=\frac{h}{f} \quad$ Bewegt sich ein Filmpurkt um den Winkel $2 w$ dureh das Bildfenster hin- 
durch, so darf sich nach dem Reflexionsgesetze der Spiegel nur um den Winkel $w$ drehen; dieser Winkel $w$ ist gleich dem Zentriwinkel des Vielecks. Die Anzahl $z$ der Spiegel ergibt sich demnach als $z=\frac{360^{\circ}}{w}$.

Wie die katoptrischen Ausgleichssysteme, so suchen auch die dioptrischen durch Einwirkung auf den abbildenden Strahlengang die mechanische Filmbewegung optisch zu kompensieren. Gelingt das dort durch zweckmäßige Beǹtzung des Reflexionsgesetzes, so geiten hier die bei der Brechung auftretenden GesetzmäBigkeiten. Das Prinzip solcher auf Strahlenbrechung beruhender Anordnungen wird in einfachster Form verwirklicht durch die rotiereade Plamparallelplatte. Fig. 22 zeigt schematisch, wie die Wanderung des Filmpunktes $A$ nach $A^{\prime}$ durch Drehung der Platte aufgehoben wird. Bezeichnet man mit $\varphi$ den Winkel, um den diese aus der zum Film parallelen Lago gedreht werden muß, um den Ausgleich für $A A^{\prime}=a$ herbeizuführen, ist fermer $n$ der Brechungsexponent und $d_{\text {.. }}$ die Dicke der Platte, so ergibt sich mit Hilfe des Gesetzes von Snellius die Beziehung:

$$
a=d \frac{\sin (\varphi-\psi)}{\cos \vartheta}
$$

$$
\text { wobei } \quad \sin \vartheta=\frac{\sin \varphi}{n}
$$

ist. Statt der einfachen Platte sind eine grobe Reihe dioptrischer Ausgleichssysteme angegeben worden, z. B. ein gleichseitiges, um die Achse drehbares Glasprisma gerader Seitenzahl, rotierende Linsenkränze u. a.

Die Vorteile, die durch Kinoapparate mit stetiger Bildbandführung gegenüber denen mit ruckweisem Weiterschalten der Bilder erreicht werden sollen, seien hier kurz zusammengefaßt. Durch die in allen seinen Teilen mit gleichförmiger Geschwindigkeit erfolgende Bewegung des Films wird seine Festigkeit nur so wenig beansprucht, daß man, wenn wänschenswert, für die Aufinahme wie für die Vorführung eine viel höhere Bildwechselfrequenz wie etwa $20-30$ in Anwendung bringen kann. Andererseits gestattet der Projektor, da ja der Wechsel von hell und dunkel fortfällt, einmal das Licht besser auszunutzen und ferner auch schon bei Anwendung einer Bildwechselfrequenz von 10 und weniger. eine flimmerfreie Projektion zu erzielen, so daß in passenden Fällen sehr an Film gespart werden kann. Trotz dieser theoretisch möglichen Vorteile haben sich die Apparate mit optischem Ausgleich der Bildwanderung noch nicht einzuführen vermocht, da die bisher vorliegenden Ausführungsformen in ihrer Leistungsfähigkeit meist noeh recht viel zu wünschen übrig lassen.

Ihrer verschiedenen Verwendung nach unterscheiden sich die Apparate für die Aufnahme von denen für die Wiedergabe in vielen Punkten. Haben jene-im wesentlichen die Aufgabe einer photographischen Kammer zu erfüllen, so sind diese den besonderen Verhältnissen angepabte Projektionsapparate. Da man beispielsweise für den Aufnahmefilm keine physiologische Verschmelzung zu erzielen braucht, ist bei den Aufnahmeapparaten eine mehrflüglige Blende $z u$ überzähligen Abblendungen des Objektivs ebensó unnötig, wie etwa die Herbeiführung einer im Verhältnis zur Ruhezeit möglichst kurzen Transportzeit des Films.

Die weiteren Einrichtungen und Methoden, die zur Gewinnung und zur Wiedergabe der Filmbilder dienen, sind der allgemeinen Technik der Photographie bzw. Projektion entlehnt, gegebenenfalls mit zweckentsprechenden Modifikationen. Thre nähere Erörterung kann daher unterbleiben, da es uns hier nur darauf ankommt, die spezifischen Eigenschaften der kinematographischen Apparate kurz zu besprechen, ohne uns auf Einzelheiten einzulassen. Ebenfalls verzichten wir auf eine Darstellung "der Kinematographie in natürlichen Farben , und mit stereoskopischem Effekt: Auch die zahlreichen Anwendungen der Kinematographie in Wissen schaft und Technik und die dabei auftretenden Probleme können in diesem Aufsatz, der nur einen allgemeinen Utberblick über die Grundlagen der Kinematographie geben soll, keinen Platz finden.

\section{Quantitatives über Kathodenstrahlen aller Geschwindigkeiten.}

Von Prof. Dr. R. Seeliger, Greifswald.

$P$. Lenard hat kürzlich in Buchform eine ausgezeichnete, von tiefster Sachkenntnis getragene Monographie ,Quantitatives über Kathodenstrahlen aller Geschwindigkeiten" veröffentlicht"). Er fabt darin die Ergebnisse seiner und seiner Schüler über nunmehx fast 3 Jahrzehnte sich erstreckenden systematischen Untersuchungen mit einer Bearbeitung der gesamten Literatur zu einem Werk von fundamentaler Bedeutung zusammen, das jeder auf diesem und auf verwandten Gebieten selbständig Forsehende mit Freude begrüBen wird, denn es gibt zum erstenmal in wirklich kritischer und zuverlässiger Weise in den verschiedensten Teilen der Physik vielgebrauchtes Material an die Hand. Auf den Inhalt - der durchaus nicht etwa populär, sondern streng' wissenschaftlich und mit. Nutzen auch für den mit dem Thema bereits Vertrauten nur in hingehendom Studium $z$ lesen ist - werde ich später noch im einzelnen eingehen; zunächst mag es gestattet sein, einige Bemerkungen allgemeiner Art vorauszuschicken. Denn hinter der rein fachlichen Bedeutung dieses Buches scheint mir noch etwas anderes $z u$ stehen, das zu manchen ermsten Gedanken anregt, weil es für die moderne Entwicklung der Wissenschaft symptomatisch ist.

1) Abhandlg, der Heidelbeng. Akad. Nr. 5, 1918. 266 S. mit 7 Kurventafeln and 4 Textfiguren. (C. Winters Univensitätsbuchhandlung 1918.) 